\title{
Epigenetics and nutrition
}

\begin{abstract}
In the last few years, the idea of food and nutrition has undergone radical changes. The paradigm defining food as a simple source of energy and body mass has evolved in a novel concept, in which nutrients can exert specific functions directly linked to human health. Several edibles contain biological active compounds that influence cellular, metabolic and physiologic processes. A variety of dietary compounds have been associated with specific effects and mechanisms of action, often involving epigenetic modifications and gene expression changes. DNA methylation, histone modifications and the activity of non-coding RNAs control chromatin condensation state, allowing the interaction of DNA with transcription factors required for transcriptional activation. Some metabolites act as substrates of key chromatin remodeling factors or compete with other substrates, influencing their catalytic activity. The quantity and quality of macronutrients assumed by diet offers a possible mechanism of interaction between the body and its environment. A number of biological compounds are known to interact with the epigenome. Folic acid, methionine, choline and other B group vitamins are an important source of one-carbon groups required for methylation of histone proteins and non-histone chromatin remodeling factors. Other compounds like polyphenols, including resveratrol, curcumin and quercetin exert a multitude of biological activities. Furthermore, these compounds can influence DNA methyltransferases, the enzymes responsible for DNA methylation. Dietary interventions aimed to maximize potential health benefits derived from nutrition have shown that dietary regimens can be very effective for prevention and treatment of several diseases. Calorie restriction, protein restriction, fast mimicking diets or time restricted feeding have shown to have significant effects on health. Food quality is crucial for the activity of biological compounds and their potential health benefits. Diets rich in animal-derived proteins are associated with higher rates of mortality compared to diets based on plant-derived proteins.
\end{abstract}

Keywords: personalized nutrition, functional foods, disease prevention, disease treatment, diet
Volume 6 Issue I - 2018

\author{
Andrea Raganelli,' Roberta Della Bona,' \\ Anna Maria Martino,' Caterina Calò,' \\ Gabriele Palozzi, ${ }^{2}$ Marco Rebecchi,' Stefano \\ Lino,' Leonardo Calò' \\ 'Division of Cardiology, Policlinico Casilino, Italy \\ ${ }^{2}$ Department of Management \& Law, University of Rome Tor \\ Vergata, Italy
}

Correspondence: Andrea Raganelli, P.zza Antonio Spaziani n ${ }^{\circ} 7$ 00030, Genazzano (Rome), Italy, Tel +39 3389001060 , Email andrea.raganelli@gmail.com Received: November 30, 2017 | Published: January 12,
2018

\begin{abstract}
Abbreviations: IGF-1, insulin-like growth factor 1; ncRNAs, non-coding RNAs; HAT, histone acetyltransferase; HDAC, histone deacetylase; DNMT1, DNA methyltransferase 1, ROS, reacting oxygen species; AdoMet or SAM, S-adenosylmethionine; AdoHcy, S-Adenosylhomocysteine; LC-PUFAs, long chain polyunsaturated fatty acids
\end{abstract}

\section{Introduction}

Epigenetics refers to the study of heritable changes in gene expression that do not depend on the DNA sequence itself. Those changes alter the phenotype without any change in the genotype and can be influenced by several factors. The environment, the way of living, habits, age, parental nutritional state during gestation and others are amongst the major factors influencing chromatin remodeling. In eukaryotes, the DNA is organized around histone proteins to form chromatin fibers. Chromatin undergoes substantial changes during cell cycle from extremely compacted, during mitosis, to stages when individual chromosomes are not visible and the chromatin appears diffuse and unorganized. Condensed chromatin is associated with transcriptional inactivity, while diffuse and relaxed chromatin fibers are correlated with active transcription. Chromatin condensation affects the accessibility of the DNA to transcription factors and other regulators preventing transcriptional activity. Epigenetic modifications controlling chromatin condensation include DNA methylation, histone modifications, alterations of chromatin structure due to chromatin remodeling and non-coding RNAs (ncRNAs). ${ }^{1}$ The acquired epigenetic changes can be inherited during cell division and result in a permanent change, maintained in the cell lineage. ${ }^{2}$

DNA methylation refers to the addition of a methyl group $\left(\mathrm{CH}_{3}\right)$ to the cytosine or adenine bases of the DNA. Adenine methylation has received very little attention so far while cytosine methylation is more characterized. Methylation of cysteine occurs to position 5 on the pyrimidine ring in a cytosine-guanine (CG) pair also known as $\mathrm{CpG}$ site. DNA methylation is achieved through the activity of DNA methyltransferase enzymes, which catalyze the transfer of methyl groups to DNA. The lack of regulation in $\mathrm{CpG}$ methylation is associated with the loss of imprinting control, the demethylation of promoters and the transcription of genes that would otherwise be silenced with the subsequent development of cancer. ${ }^{3}$

\section{Histone modifications}

The nucleosome is the fundamental subunit of the chromatin. It consists of 147 base pairs of double stranded DNA wound twice around an octameric histone protein complex. ${ }^{4}$ Several histone modifications have been described, including mono-, di-, and trimethylation, acetylation, ubiquitylation, phosphorylation, and ribosylation. Histone modifications most commonly occur on the $\mathrm{N}$ terminus of histone tails. Modifications on histone tails can influence 
the chromatin state and control the accessibility of the DNA to transcription factors. Different modifications are associated with active or inactive DNA transcription and only rarely one specific histone modification determines gene expression levels. It is the combination of specific histones and types of modification that determines the active or inactive state of chromatin. Histone acetyl transferases (HAT) are the enzymes responsible for acetylation, the addition of acetyl group. Histone deacetylases (HDACs) are responsible for the removal of acetyl groups, referred to as deacetylation. Several genes associated with histone modifications are commonly overexpressed in prostate cancer ${ }^{5}$ and aberrant patterns of histone modifications have been associated with carcinogenesis. ${ }^{6}$

\section{Non-coding RNAs (ncRNAs)}

These are untranslated transcripts that exert functional roles in epigenetic gene regulation. On the base of their length, they can be classified as short (sncRNAs less than 200 nucleotides), or long (lncRNAs longer than 200 nucleotides) and act in regulating gene expression by a number of mechanisms, including heterochromatin formation and inhibition of translation. ${ }^{3,7}$ There is a strong association between misregulation of miRNAs activity and the development of cancer. For example miR-101 and other factors targeting the histone methyl-transferase EZH2 are associated with carcinogenesis (Figure 1). ${ }^{8,9}$

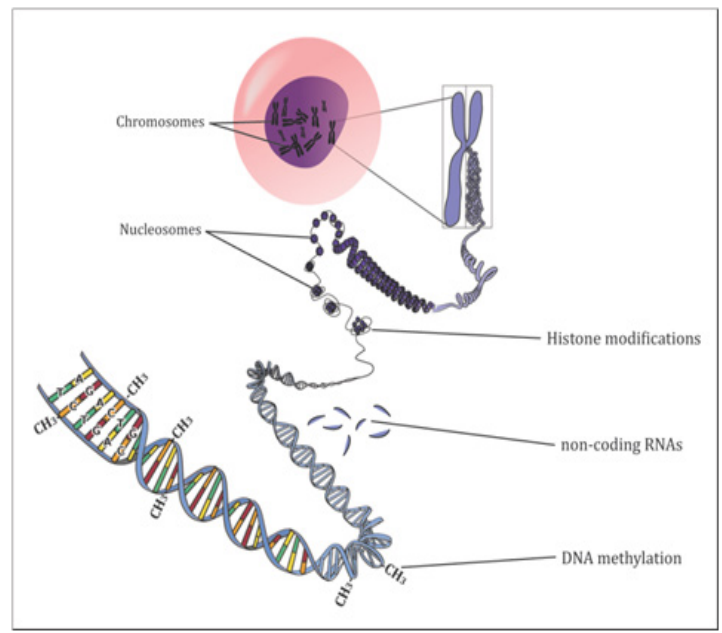

Figure I Principal epigenetic mechanisms regulating gene expression.

The extension of life expectancy in developed countries paves the way for the development of older populations and the corresponding increase of age-related diseases. Metabolic disorders, diabetes, heart and lung diseases, cancers and other diseases have been associated with several genetic and environmental (diet, pollution, stress) factors. A variety of factors are known to influence gene expression (Table 1). The genetic background characterizes every single individual. However, we cannot directly manipulate our genome to improve our health. Knowing our characteristics we can indeed act on the external factors to ameliorate life quality and health. The beneficial effects of foods have been known for a long time. Ancient Egyptians, Romans and Chinese already used honey to cure wounds or intestinal problems. ${ }^{2}$ Nowadays, we have a growing collection of data indicating specific links between nutrients, metabolism, health and ageing. In the last decade, epigenetic modifications become central in cancer development and ageing. Several phytochemicals, other bioactive food components and metabolites can modulate oxidative stress, inflammatory and metabolic pathways influencing chromatin remodeling. ${ }^{10}$ A variety of dietary nutrients can interact with DNA methylation enzymes and histone modifications or alter the availability of substrates required for enzymatic reactions. ${ }^{11}$ Epigenetics appears to be the link between environment-associated diseases, nutrition and health. Nutrients can modify the epigenetic program and influence gene expression therefore controlling the intake of functional food components through diet can be powerful in preventing age-related and other diseases including cancer. ${ }^{12-14}$ It is emerging a novel view of food and metabolites, in contraposition to the classical paradigm based on the chemical conversion of food in energy and body mass. Foods and metabolites can actually impact on the genome through epigenetic regulation and control of stress adaptive responses, energy metabolism, immune homeostasis, and physiology. ${ }^{15-18}$ Furthermore, studies in animal models suggest that maternal nutritional imbalance, during critical period of development (i.e. gestation), may influence the health of the offspring and perhaps even next generation's health. Diabetes, cardiovascular disease, allergy, some forms of cancer, cognitive decline, and affective disorders may have important connection with the parental nutritional status. ${ }^{19-25}$ Studies in human populations following famine reflect the results on animal models, suggesting that nutritional insult during pregnancy may increase the probability of cardiovascular disease development in the offspring. ${ }^{26-28}$ Paternal nutrition may also play an important role in offspring and future generations health. ${ }^{29,30}$ All these studies converge on the central role exerted by nutrition during the entire life, and perhaps even through generations, suggesting that diet could be used as a pharmacological agent to improve health and life expectancy.

Table I Principal factors influencing gene expression

\begin{tabular}{l}
\hline Factors influencing gene expression \\
\hline Genetic Background \\
Nutrients Levels \\
Dietary Components (fats, proteins, phytoestrogens, etc) \\
Toxins (chemicals, metals, drugs, etc ) \\
Physical exercise and activity \\
Life style (sleep, work, etc) \\
Sunlight exposure and vitamin D levels \\
Interactions with bacteria and viruses \\
Further unknown factors
\end{tabular}

\section{Discussion}

Some food components have been described to interact with the epigenome in different ways. For example, folic acid, B vitamins and others are key components of a metabolic pathway generating methyl groups available for DNA methylation enzymes. The S-adenosylmethionine (AdoMet or SAM) and the S-adenosylhomocysteine (AdoHcy) metabolites participate in the onecarbon metabolism, are involved in the transfer of methyl groups and in the inhibition of methyl transferases, respectively. Thus, AdoMet and AdoHcy can alter DNA methylation and histone modifications. Other foods contain anti-oxidant metabolites, which counteract reacting oxygen species (ROS) responsible for the oxidative stress influencing DNA demethylation. ${ }^{31-35}$ Excessive formation of ROS and oxidative 
stress can lead to pathological conditions, including cancer. ${ }^{36,37}$ Folate, also known as vitamin B9, is a one-carbon donor and participates to the one-carbon metabolism. Folate is essential for the synthesis of DNA, proteins and phospholipids, cell division, and the formation of erythrocytes. Folate has been associated with atherosclerosis, neurological disorders, and carcinogenesis. ${ }^{38}$ Furthermore it exerts antioxidant activity influencing the NO synthase enzyme. ${ }^{39}$ Folate is acquired exclusively from diet, and deficiency in folate affects human development and health. A lack of folate during pregnancy could lead to abnormal neural tube development in the baby, while in adults, it could lead to megaloblastic anemia, the release of immature erythrocytes into the blood stream. Other vitamins B play a central role in one-carbon metabolism. Methionine is one of the essential amino acids that may interfere with one-carbon metabolism, and act on the epigenome to modulate gene expression. Polyphenols represents one of the most abundant classes of natural products obtained from plants. In addition to their natural variability, most of polyphenols can be glycosylated or acetylated at distinct positions of the sugar skeleton originating an innumerable number of different molecules. ${ }^{40}$ They can be classified in phenolic acids (benzoic acids and cinnamic acids), flavonoids (antocyanins, flavan-3-ols, flavones, flavanones and flavonols), polyphenolic amides (capsaicinoids and avenanthramides) and other polyphenols (resveratrol, ellagic acid, lignans, curcumin, rosmarinic acid, tannins). ${ }^{40}$ Polyphenols exert a wide range of biological activities, including potential effects against cancer, aging, and inflammation. ${ }^{41}$ Besides, they have strong antioxidant activity and can influence DNA methylation, by inhibiting the enzyme DNMT1. ${ }^{3}$ Epigallocatechin gallate (EGCG) is the ester of epigallocatechin and gallic acid, and is the most abundant catechin found in tea. Polyphenol compounds act as powerful antioxidant ${ }^{42}$ and show effective anticancer properties. EGCG in particular was described alter the epigenome by inhibiting the enzyme DNA methyl transferases in human esophageal, colon, prostate, and mammary cancer cell lines. ${ }^{43}$ Moreover, EGCG was correlated with the inhibition of invasive metastasis in pancreatic cancer, through the inhibition of HDAC enzymes and increased expression of the histone H3 ${ }^{44}$ Curcumin from turmeric (Curcuma longa, Curcuma domestica) is another member of the polyphenol class with strong anticancer potential, which was shown to influence histone modifications. In particular, it was associated with the induction of histone hypoacetylation in glioma cells ${ }^{45}$ and the inhibition of H3K27 methylation mediated by EZH2 (histone-lysine $\mathrm{N}$-methyltransferase) in metastatic cancer cells. ${ }^{46}$ In addition, extracts from Curcuma domestica have been reported as effective as ibuprofen for treatments of knee osteoarthritis. ${ }^{47}$ Quercetin is a further flavonoid with a wide range of biological properties. Recent studies have associated quercetin to strong anti-inflammatory, anti-viral and anticarcinogenic activities along with attenuation of lipid peroxidation. ${ }^{48}$ Moreover, this polyphenol was shown to act as mast cell inhibitor to treat allergic and inflammatory diseases. ${ }^{49}$ Thus, polyphenols can act on the epigenome through different mechanisms, including DNA methylation and histone modifications. Other vitamins can influence health by interacting with the epigenome. Retinoic acid (vitamin A) is required for growth and development. This vitamin interacts with a number of the Polycomb group proteins, responsible of chromatin remodeling mediating the epigenetic regulation of several genes. ${ }^{3}$ Vitamin $D$ also interacts with the epigenome in different ways. Key genes of the vitamin D pathway are regulated by DNA methylation of $\mathrm{CpG}$ sites, located in the promoter of those genes. Furthermore, the vitamin D receptor protein directly interacts with a number of cofactors responsible for the activation and repression of several chromatin remodeling factors, including histone acetyltransferases, histone deacetylases, and histone methyltransferases. ${ }^{50}$ Fatty acids are carboxylic acids with a saturated or unsaturated aliphatic chain that can play a variety of different functions, from energy sources to membranes constitution and maintenance. They can influence the membrane structure and function, impacting on signaling pathways, transcription factor activity, and ultimately gene expression. Thus, fatty acids can influence the activity, the signal response and metabolism of cells and tissues playing health-promoting roles. Fatty acids have an effect on a variety of diseases, encompassing metabolic diseases, diabetes, cardiovascular diseases and cancer. ${ }^{51}$ Omega- 3 and omega- 6 long chain polyunsaturated fatty acids (LC-PUFAs), including new families of PUFA-derived lipid mediators called resolvins, protectins and maresins, play active anti-inflammatory roles and are crucial for the nervous system functionality. Many other biological compounds are known to have the ability to influence chromatin-remodeling factors. Allyl compounds, isothiocyanates, lycopene, hydroxytyrosol can exert antioxidant and other health promoting activities.

An increasing number of studies highlight new connections between health and nutrition, leading the development of a range of dietary interventions to treat diverse diseases. The study of intertwined interactions between diet and genome has led to the development of nutrigenetics and nutrigenomics. This novel field aims to shed new light on the interactions between epigenetics, nutrigenetics, transcriptomics, metabolomics, and lipidomics on a whole genome scale. Aging is the result of complex processes, involving the accumulation throughout life of cellular damages that progressively impair cellular functions and increase the risk of disease and death. ${ }^{52}$ Several studies focusing the link between nutrition and aging described, initially in animal models and subsequently in humans, the beneficial effects of fasting periods to counteract the aging process and extend life expectancy. Therefore, dietary interventions aiming to counteract processes such as aging as well as a variety of metabolic and other diseases have started to gather the attention of a larger audience. Public interest on food quality and food nutritional content is growing everyday and the market mirrors an increasing request of specific bioactive-containing foods with distinct properties. The increasing number of diseases associated with animal-derived foods containing low quality fatty acids and the lack of physical activity is forcing doctors to identify novel strategies beyond pharmaceutical treatments to prevent, reverse or cure diseases (Figure 2). Dietary restriction is used to describe a variety of diets characterized by specific nutrient and/or energy restriction, associated with positive effects on health. ${ }^{53}$ In particular, the reduction of total food intake (between $20 \%$ and $40 \%$ ) is referred to as calorie restriction. ${ }^{54}$ Protein restriction indicates a reduction of total protein intake, while methionine or tryptophan restriction refers to the restriction of the methionine or tryptophan amino acid specifically. These dietary interventions have been associated with cardiovascular disease prevention and treatment. The restriction of total energy intake is not necessarily required to achieve beneficial effects derived by calorie restriction. Protein restriction, fast mimicking diets and time restricted feeding can trigger similar beneficial health responses. Dietary regimens based on macronutrient restriction without calorie restriction can be effective in counteracting metabolic diseases. Protein restriction has received an increasing attention, and the quality of protein and amino acids composition may play a crucial role metabolic disease prevention and treatment. Individual amino acids, the building blocks of protein, can have distinct effects on metabolism. Decreased intake of branched chain amino acids, such as leucine, isoleucine and 
valine, leads to a reduction of glucose and IGF-1 levels, improving metabolism. ${ }^{53}$ Other dietary strategies focus on the beneficial effect of fasting. Fasting periods activate AMPK, which promotes catabolic and repair processes, and inhibits the activity of mTOR and anabolic pathways. ${ }^{55}$ Among these strategies we would like to mention time restricted feeding and fast mimicking diets. Time restricted feeding is characterized by the standard caloric intake consumed within few hours, to allow a fasting period of 12-14hours a day. Fast mimicking diets are designed to provide macronutrient content able to mimic water only fasting, while providing complete micronutrient content. For example, a human fast mimicking diet can be characterized by a five-day regimen, repeated 3 cycles in a month, providing between 725 and $1090 \mathrm{kcal}$ per day. ${ }^{55}$ The Mediterranean diet is associated with a lower risk of cardiovascular and cancer disease development, as well as with prolonged lifespan. ${ }^{56,57}$ It is based on daily intakes of high quantities of fresh fruit and vegetables, olive oil, cereals and fresh fish. The beneficial effects of the Mediterranean diet may be the result of the combined action of high antioxidant-containing meals, enrichment of olive oil derived fatty acids and daily life activities promoting social interactions and positive moods. A variety of dietary interventions proved to be effective in counteracting disease development and may be a significant support for chronic diseases treatment. Dietary interventions based of meal composition without calorie restriction can be specifically designed to prevent or treat agerelated, metabolic, and other diseases. Selected functional foods with high bioactive compounds content can be used to control diseaseassociated markers, and dietary regimens can be planned based on specific food properties. Genetic analyses allow the identification of variants associated with metabolic or other diseases that may interact with nutrients in order to identify foods "to avoid" or "to prefer" on the base of our unique characteristics (Figure 3). Personalized dietary interventions will soon allow medical doctors to use nutrition as a primary tool for medical treatments.

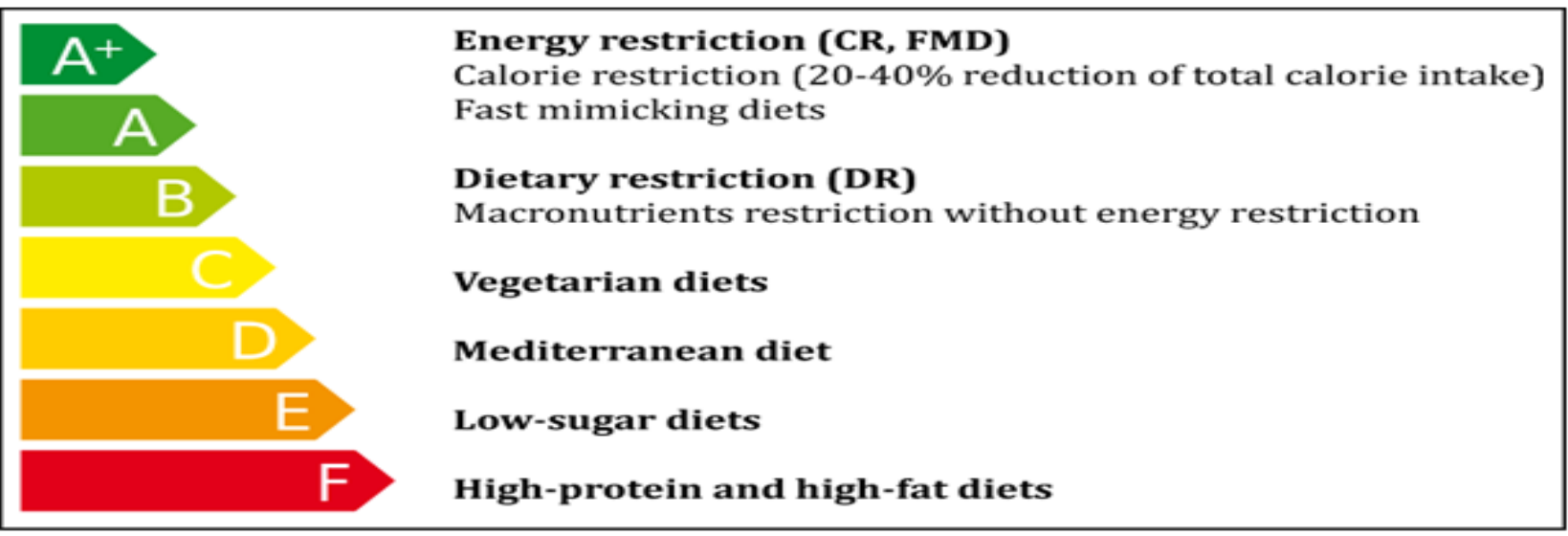

Figure 2 Comparison of diets based on the total intake of calories. (CR: calorie restriction; FMD: fast mimicking diets; DR: dietary restriction).

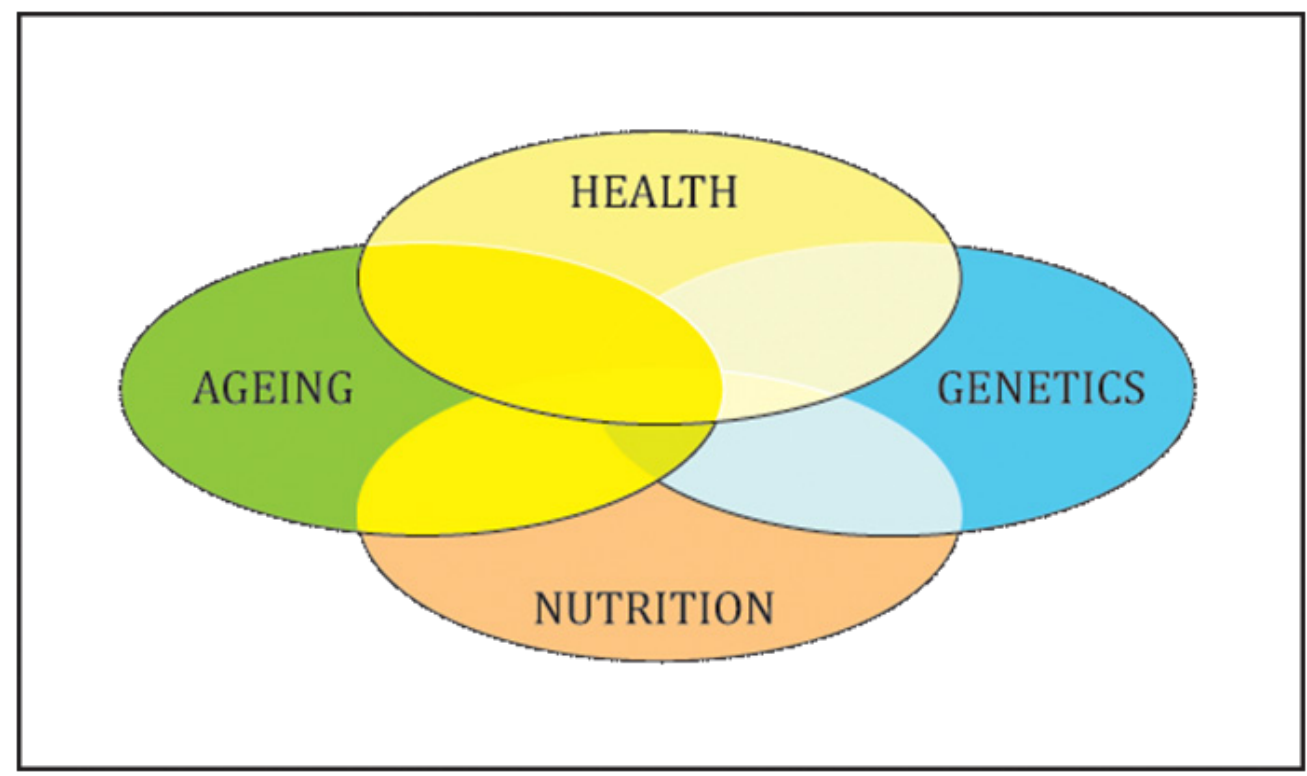

Figure 3:The intertwined interactions between genetics, nutrition, ageing and health will be crucial for the development of personalized nutritional interventions. 


\section{Conclusion}

Many edibles contain biological compounds that play a variety of crucial roles in metabolic and cellular processes. These biological compounds are critical in promoting health and counteract the development of a variety of diseases. Polyphenols, fatty acids, vitamins, amino acids, and other metabolites can interact with the epigenome and exert a variety of activities required for cellular processes directly linked to health. Dietary interventions can have significative effects on cardiovascular diseases, metabolic diseases, and cancer. Effects of dietary interventions can be measured using specific markers associated with distinct pathologies. A dietary regimen based on high antioxidant intake with meals rich in fruit and vegetables is crucial for maintaining proper health functions, and prevent precocious ageing. The Mediterranean diet based on high daily consumption of fresh fruit and vegetables, olive oil, fresh fish and cereals is associated with lower risk of cardiovascular and cancer disease development. Olive oil and unsaturated fatty acids should be preferred to saturated ones while fruit and vegetables should be served within few days after gathering, to ensure high bioactive compounds content. Controlling energy intake is crucial to achieve beneficial effects of dietary interventions. Calorie restriction can be sustained for prolonged periods of time under strict medical advice and lead to significant health improvements, including a reduction of inflammatory and cardiovascular markers. Similar benefits can be obtained through diets based on dietary restriction, protein restriction or time restricted feeding. Fast mimicking diets have also shown to be effective in reducing diseases-associated markers, including blood pressure and heart rate. It is already possible to analyze genetic differences among individuals in order to characterize metabolic and other genetic peculiarities to identify personalized dietary interventions with therapeutic or beneficial effects. The development of analytic methods for the characterization of individual responses to nutrients and the identification of functional foods enriched in bioactive compounds will allow more accurate personalized nutritional strategies in the next future.

\section{Acknowledgements}

None.

\section{Conflict of interest}

The author declares no conflict of interest.

\section{References}

1. James RT, Victoria VL. Epigenetics Judge, jury and executioner of stem cell fate. Epigenetics. 2012;7(8):823-840.

2. Sang Woon C, Simonetta F. Epigenetics: a new bridge between nutrition and health. american society for nutrition. Adv Nutr. 2010;1:8-16.

3. Karen SB, Lynnette RF. The interaction between epigenetics, nutrition and the development of cancer. Nutrients. 2015;7(2):922-947.

4. Davey CA, Sargent DF, Luger K, et al. Solvent mediated interactions in the structure of the nucleosome core particle at 1.9 a resolution. $J$ Mol Biol. 2002;319(5):1097-1113.

5. Bianco MT, Chiam K, Buchanan G, et al. Global levels of specific histone modifications and an epigenetic gene signature predict prostate cancer progression and development. Cancer Epidemiol Biomark Prev. 2010;19(10):2611-2622
6. Sawan C, Herceg Z. Histone modifications and cancer. Adv Genet. 2010;70:57-85.

7. Veronica JP, Claes W. Non-coding RNAs as direct and indirect modulators of epigenetic regulation. Epigenetics. 2014;9(1):3-12.

8. Varambally S, Cao Q, Mani RS, et al. Genomic loss of microRNA-101 leads to overexpression of histone methyltransferase EZH2 in cancer. Science. 2008;322(5908):1695-1699.

9. Friedman JM, Liang G, Liu CC, et al. The putative tumor suppressor microRNA-101 modulates the cancer epigenome by repressing the polycomb group protein EZH2. Cancer Res. 2009;69(6):2623-2629.

10. Wang L, Chia NC, Lu X, et al. Hypothesis: environmental regulation of 5-hydroxymethylcytosine by oxidative stress. Epigenetics. 2011;6(7):853856.

11. Stanger O. Physiology of folic acid in health and disease. Curr Drug Metab. 2002;3(2):211-223.

12. Bachmeier BE, Mirisola V, Romeo F, et al. Reference profile correlation reveals estrogen-like transcriptional activity of Curcumin. Cell Physio Biochem. 2010;26(3):471-482.

13. Senft C, Polacin M, Priester M, et al. The nontoxic natural compound Curcumin exerts anti-proliferative, anti-migratory, and anti-invasive properties against malignant gliomas. BMC Cancer. 2010;10:491.

14. Al Jabri AA. Honey, milk and antibiotics. Afr $J$ Biotechnol. 2005;4(13):1580-1587.

15. Vel Szic KS, Ndlovu MN, Haegeman G, et al. Nature or nurture: let food be your epigenetic medicine in chronic inflammatory disorders. Biochem Pharmacol. 2010;80(12):1816-1832.

16. Miceli M, Bontempo P, Nebbioso A, et al. Natural compounds in epigenetics: a current view. Food Chem Toxicol. 2014;73:71-83.

17. Remely M, Lovrecic L, de la Garza AL, et al. Therapeutic perspectives of epigenetically active nutrients. Br J Pharmacol. 2015;172(11):2756-2768.

18. Gluckman PD, Hanson MA, Cooper C, et al. Effect of in utero and earlylife conditions on adult health and disease. $N$ Engl J Med. 2008;359(1):6173.

19. Burdge GC, Lillycrop KA. Nutrition, epigenetics, and developmental plasticity: implications for understanding human disease. Annu Rev Nutr. 2010;30:315-339.

20. Anway MD, Cupp AS, Uzumcu M, et al. Epigenetic transgenerational actions of endocrine disruptors and male fertility. Science. 2005;308(5727):1466-1469.

21. Anway MD, Skinner MK. Epigenetic transgenerational actions of endocrine disruptors. Endocrinology. 2006;147(6 Suppl):S43-49.

22. Jirtle RL, Skinner MK. Environmental epigenomics and disease susceptibility. Nat Rev Genet. 2007;8(4):253-262.

23. Barker DJ, Martyn CN. The maternal and fetal origins of cardiovascular disease. J Epidemiol Community Health. 1992;46(1):8-11.

24. Jackson AA, Burdge GC, Lillycrop KA. Diet, nutrition and modulation of genomic expression in fetal origins of adult disease. World Rev Nutr Diet. 2010;(101):56-72.

25. Painter RC, Osmond C, Gluckman P, et al. Transgenerational effects of prenatal exposure to the Dutch famine on neonatal adiposity and health in later life. BJOG. 2008;115(10):1243-1249.

26. Lumey LH, Stein AD. Transgenerational effects of prenatal exposure to the Dutch famine. BJOG. 2009;116(6):868. 
27. Roseboom T, de Rooij S, Painter R. The Dutch famine and its long-term consequences for adult health. Early Hum Dev. 2006;82(8):485-491.

28. Whitelaw E. Epigenetics: sins of the fathers, and their fathers. Eur J Hum Genet. 2006;14(2):131-132.

29. Kaati G, Bygren LO, Edvinsson S. Cardiovascular and diabetes mortality determined by nutrition during parents' and grandparents' slow growth period. Eur J Hum Genet. 2000;10(11):682-688.

30. Ferreira CV, Justo GZ, Souza AC, et al. Natural compounds as a source of protein tyrosine phosphatase inhibitors: application to the rational design of small-molecule derivatives. Biochimie. 2006;88(12):1859-1873.

31. Perillo B, Ombra MN, Bertoni A, et al. DNA oxidation as triggered by H3K9me2 demethylation drives estrogen-induced gene expression. Science. 2008;319(5860):202-206.

32. Chouliaras L, van den Hove DL, Kenis G, et al. Age-related increase in levels of 5-hydroxymethylcytosine in mouse hippocampus is prevented by caloric restriction. Curr Alzheimer Res. 2012;9(5):536-544.

33. Valinluck V, Sowers LC. Endogenous cytosine damage products alter the site selectivity of human DNA maintenance methyltransferase DNMT1. Cancer Res. 2007;67(3):946-950.

34. Langie SA, Kowalczyk P, Tomaszewski B, et al. Redox and epigenetic regulation of the APE1 gene in the hippocampus of piglets: the effect of early life exposures. DNA Repair (Amst). 2014;18:52-62.

35. Fraga MF, Esteller M. Epigenetics and aging: the targets and the marks. Trends Gene. 2007; 23(8):413-418.

36. Lawless MW, Obyrne KJ, Gray SG. Targeting oxidative stress in cancer Expert Opin Ther Targets. 2010;14(11):1225-1245.

37. Rong T. Chemistry and biochemistry of dietary polyphenols. Nutrients. 2010;2(12):1231-1246.

38. Stanger O, Wonisch W. Enzymatic and non-enzymatic antioxidative effects of folic acid and its reduced derivatives. Subcell Biochem. 2012;56:131161.

39. Olivia SA, Karilyn ES, Dana CD. Nutrition and epigenetics. An interplay of dietary methyl donors, one-carbon metabolism, and DNA methylation. J Nutr Biochem. 2012;23(8):853-859.

40. Fang M, Chen D, Yang C. Dietary polyphenols may affect DNA methylation. J Nutr. 2007;137(1 Suppl):223S-228.

41. Matteo T, Valentina $Z$, Alessandro F, et al. The aging process and potential interventions to extend life expectancy. Clinical Interventions in Aging. 2007;2(3):401-412.

42. Fuchs-Tarlovsky V. Role of antioxidants in cancer therapy. Nutrition. 2003;29(1):15-21.
43. Craciunescu CN, Johnson AR, Zeisel SH. Dietary choline reverses some, but not all, effects of folate deficiency on neurogenesis and apoptosis in fetal mouse brain. The Journal of Nutrition. 2010;140(6):1162-1166.

44. Margie TB, Brian CS, John MD. Mechanism of human SIRT1 activation by resveratrol. THE Journal of biological chemistry. 2005;280:17187-17195.

45. Hua WF, Fu YS, Liao YJ, et al. Curcumin induces down-regulation of EZH2 expression through the MAPK pathway in MDA-MB-435 human breast cancer cells. Eur J Pharmacol. 2010;637(1-3):16-21.

46. Dimri M, Bommi PV, Sahasrabuddhe AA, et al. Dietary omega-3 polyunsaturated fatty acids suppress expression of EZH2 in breast cancer cells. Carcinogenesis. 2010;31(3):489-495.

47. Hamed M, Stefano Di B, Valter DL. Dietary interventions, cardiovascular aging, and disease animal models and human studies. Circ Res. 2016;118(10):1612-1625.

48. Zuyi W, Bodi Z, Shahrzad A, et al. Quercetin is more effective than cromolyn in blocking human mast cell cytokine release and inhibits contact dermatitis and photosensitivity in humans. PLOS ONE. 2012;7(3):e33805.

49. Naghma K, Deeba NS, Nihal A, et al. Fisetin: A dietary antioxidant for health promotion. Antioxidants \& Redox Signaling. 2013;19(2):151-162.

50. Marco C, Gaspard C, Cédric M, et al. Resveratrol stimulates the metabolic reprogramming of human $\mathrm{CD} 4+\mathrm{T}$ cells to enhance effector function. $\mathrm{Sci}$ Signal. 2017;10(501):eaal3024.

51. Rafael Z, Nabil el J, Noemi T, et al. Significance of long chain polyunsaturated fatty acids in human health. Clin Transl Med. 2017;6(1):25.

52. Yao Li, Jiaying $\mathrm{Y}$, Chunyan $\mathrm{H}$, et al. Quercetin, Inflammation and Immunity. Nutrients. 2016;8(3):167.

53. Luigi F, Nicole EC, Sebastian IAA, et al. Decreased consumption of branched chain amino acids improves metabolic health. Cell Rep. 2016;16(2):520-530.

54. Fontana L, Meyer TE, Klein S, et al. Long-term calorie restriction is highly effective in reducing the risk for atherosclerosis in humans. Proc Natl Acad Sci USA. 2004;101(17):6659-6663.

55. Valter DL, Sarchidananda P. Fasting, circadian rhythms, and time restricted feeding in healthy lifespan. Cell Metab. 2016;23(6):1048-1059.

56. Emilio R, Miguel A, Martinez G, et al. Mediterranean diet and cardiovascular health: teachings of the PREDIMED study. Adcances in Nutrition. 2014;5:330S-336S.

57. La Vecchia C. Mediterranean diet and cancer. Public Health Nutr. 2004;7(7):965-968. 